\title{
Is the cluster of galaxies "Abell 194" surrounded by an Einstein-Straus vacuole?
}

\author{
R. Plaga ${ }^{1}$ \\ Franzstr. 40, 53175 Bonn, Germany, e-mail: rainer.plaga@gmx.de \\ Received 8 June 2005 / Accepted 29 July 2005

\begin{abstract}
It is suggested that an "Einstein-Straus vacuole" - a region of space time with a metric obtained by solving the equations of general relativity of a mass condensation in an expanding universe with vanishing cosmological constant - surrounds the cluster of galaxies "Abell 194". This hypothesis is shown to predict a distribution of galaxy redshifts that is in better accord with observations than the one expected in the cosmological concordance model.
\end{abstract}

Key words. galaxies: clusters: individual: Abell 194 - cosmology: observations - gravitation

60 years ago Einstein \& Straus $(1945,1946)$ determined the metric of space-time near a star embedded in an expanding universe without a cosmological constant within general relativity (GR). They assumed the star with mass $M$ is surrounded by an empty spherical cavity with a radius:

$r_{\mathrm{ES}}=\left(\frac{3 M}{4 \pi \rho_{\mathrm{M}}}\right)^{1 / 3}$

Here $\rho_{\mathrm{M}}$ is the mean matter density of the universe. The initially homogeneous matter within the cavity can be thought to be "condensed into the star".

Einstein and Straus found that space-time in the interior of the cavity is described by a static Schwarzschild metric. The cavity boundary expands with the unimpeded Hubble flow. Outside the cavity space-time is described by the expanding Friedmann-Lemaitre metric of a homogeneous universe. The "static blop of vacuum with Schwarzschild metric" that surrounds the star has been called "Einstein-Straus vacuole" (Bonnor 1987). With a non-vanishing cosmological constant $\Lambda$ Eq. (1) remains valid, but the metric within this " $\Lambda$ vacuole" is the Schwarzschild-de Sitter metric (for a summary see Gilbert 1956). In its Newtonian limit one finds that a new force directed away from the central mass should exceed gravitational attraction beyond a radius

$r_{\mathrm{m}}=\left(\frac{3 G M}{\Lambda}\right)^{\frac{1}{3}}$

The distance from a central mass that is embedded in an expanding universe at which test particles just begin to follow the outward Hubble flow rather than being attracted towards the centre is commonly called the "turnaround radius" $r_{\text {ta }}$. In an
Einstein-Straus vacuole this transition takes place at the outer boundary of an Einstein-Straus vacuole, symbolically

$r_{\mathrm{ta}}($ Einstein - Straus vacuole $)=r_{\mathrm{ES}}$,

whereas for the $\Lambda$-vacuole outward expansion sets in at $r_{\mathrm{m}}$ and thus:

$r_{\mathrm{ta}}(\Lambda$ vacuole $)=r_{\mathrm{m}}$

For the cosmological concordance model (Spergel et al. 2003) (for which the parameters $H_{0}=70 \mathrm{~km} \mathrm{~s}^{-1} \mathrm{Mpc}^{-1}$ and $\Omega_{\mathrm{M}}=$ $0.3, \Omega_{\Lambda}=0.7$ are assumed throughout this paper) $r_{\mathrm{ES}}$ exceeds $r_{\mathrm{m}}$ by about a factor 1.7 at the present epoch. Vacuoles do not surround stars like our sun, because they are already embedded in a static space time within our Galaxy. Clusters of galaxies, among the largest known structures that are gravitationally bound, could be surrounded by vacuoles. Within the concordance model an isolated fully collapsed cluster is expected to be surrounded by a $\Lambda$ vacuole. Clusters might not have completed their collapse at the present time. This would reduce the turnaround radius as compared the value predicted in Eq. (4) and one concludes:

$r_{\text {ta }}($ galaxy cluster, today $) \leq r_{\mathrm{m}}$

The medium-compact cluster Abell 194 (A194) at a distance of about $76 \mathrm{Mpc}$ is fairly isolated and its central galaxies have approached energy equipartition. Its central region is characterised by an elongated line of bright galaxies, but its outer region is spherical (Zwicky \& Humason 1964). The redshifts of 6916 galaxies around this cluster have been collected and analyzed in the recent CAIRNS survey (Rines et al. 2003). In a scatter plot against clustercentric radius $r$ they display a distinct density enhancement around the cluster redshift $z=0.0178$ within a well defined boundary. The redshift amplitude of the 
boundary is observed to decrease with clustercentric radius $r$ from $\Delta z=z$ (boundary) $-0.0178 \approx \pm 4 \times 10^{-3}$ at $r=0$ to zero at a distance of $r_{\mathrm{c}} \approx 13.2 \mathrm{Mpc}$. This trumpet shaped "caustic" boundary (with a "mouth piece" at $r_{\mathrm{c}}$ ) had been theoretically predicted for galaxies that fall radially onto a cluster (Kaiser 1987). The pattern is smoothed out, but does not vanish, if galaxies bound to the cluster possess random motions perpendicular to the infall direction (Diaferio 1999). In theoretical calculations the dispersion of redshifts becomes minimal at the turnaround radius $r_{\mathrm{ta}}$. This is because at this radius excluding random motions - infalling or orbiting galaxies are at rest relative to the cluster and therefore have the mean cluster redshift, i.e. $\Delta z=0$. Therefore - if observationally the caustic boundary is a distinct, continuous structure - it is believed, that the galaxies do not follow the Hubble flow at clustercentric distances below $r_{\mathrm{c}}$ (Diaferio 2005). Symbolically the observations of A194 seem to indicate:

$$
r_{\mathrm{c}}(\mathrm{A} 194) \approx 13.2 \mathrm{Mpc} \leq r_{\mathrm{ta}}(\mathrm{A} 194)
$$

For an observed total cluster mass

$$
M(\mathrm{~A} 194)=(2.9 \pm 2.2) \times 10^{14} M_{\odot}
$$

(Rines et al. 2003) one finds by inserting $\Lambda=8 \pi \mathrm{G} \rho_{\Lambda}$ into Eq. (2) and restating Eq. (5):

$r_{\mathrm{m}}(\mathrm{A} 194)=6.9_{-2.6}^{+1.4} \mathrm{Mpc} \geq r_{\mathrm{ta}}(\mathrm{A} 194)$.

This inequation disagrees with the one in Eq. (6) by more than four times the stated error. The observed turnaround radius of A194 is much larger than the upper limit from Eq. (8) that would seem to be a firm prediction of the concordance model of cosmology.

One possible explanation for this discrepancy is that the observed caustic boundary around A194 is due to galaxies that are related but unbound to the cluster and which coincidentally have redshifts consistent with being in an infall pattern onto A194, i.e. the observed shape of the caustic pattern is due to chance at least beyond $r_{\mathrm{m}}$ (Rines 2005).

An alternative hypothesis, called "ES", is:

"Abell 194 is surrounded by an Einstein-Straus vacuole with a radius of about $13.2 \mathrm{Mpc}$. The mass within the vacuole has nearly completely collapsed onto the central virialized cluster. The vacuole contains a static Hernquist halo with galaxies on isotropic orbits."

Assuming $\Omega=0.3$, Eqs. (1), (7) yield:

$r_{\mathrm{ES}}(\mathrm{A} 194)=11.7_{-4.4}^{+2.4} \mathrm{Mpc}$.

If hypothesis ES is true it is plausible that the observed $r_{\mathrm{c}}$ corresponds to the turnaround radius and Eq. (3) predicts:

$r_{\mathrm{ta}}=r_{\mathrm{ES}} \approx r_{\mathrm{c}}$.

This relation is fulfilled by the numerical values of Eqs. (6), (9) within the stated 1-sigma errors. Rines et al. (2003) showed that the observed redshifts around A194 could well be due to a radial mass- and velocity-dispersion distribution expected in a static Hernquist halo. The mean halo density of A194 near $r_{\mathrm{c}}$ in the Hernquist model is about 60 times smaller than the mean density of the universe, i.e. the outer halo would be a good approximation to the "empty cavity" within the EinsteinStraus vacuole.

Hypothesis ES thus seems to describe observations well and is a solution of the GR field equations in a $\Lambda=0$ universe.

What would be the implications for the nature of $\Lambda$ in general if hypothesis ES were true? The simplest (but not only ${ }^{1}$ ) modification of the concordance model that would render possible hypothesis ES would be that the cosmological constant vanishes or at least does not act on distance scales up to about $20 \mathrm{Mpc}$. While the observational evidence for an accelerated cosmic expansion seems compelling, its physical origin is still a matter of debate. Besides a cosmological constant some negative-pressure fluid with adjustable properties ("dark energy") might be responsible. Recently Kolb et al. (2005) even proposed an acceleration mechanism that does not rely on a cosmological constant or any other "dark energy" component. Therefore a modified $\Lambda$ that does not act at least up to cluster distance scales does not seem to be in contradiction with firmly established facts. However, the case for such a far reaching conclusion from the observation of just one cluster of galaxies remains weak.

Can Einstein-Straus vacuoles form around clusters of galaxies if $\Lambda=0$ ? Even with a vanishing $\Lambda$ the idealized spherical-infall model of cluster formation predicts a turnaround radius for A194 at the present time of $r_{\mathrm{ta}}$ (spherical infall, A194) $\approx 4.8 \mathrm{Mpc}$ (Rines et al. 2003), which is far smaller than $r_{\mathrm{ES}}(\mathrm{A} 194)$. However, more realistic simulations (e.g. Diaferio 1999) show that cluster formation occurs very anisotropically and involves effects like shell crossings not taken into account in the spherical-infall model. Therefore $r_{\mathrm{ta}}$ (spherical infall) should only be considered as an indicative but not quantitative estimate (Diaferio 2005). Other modifications of the concordance model than a vanishing $\Lambda$ would have an unknown influence on the collapse process. If some fraction of clusters of galaxies is expected to have completed their collapse by now - as required by hypothesis ES - remains unclear presently.

Further observations of complete caustics around relaxed, isolated clusters of galaxies could test hypothesis ES. E.g. the Coma cluster observationally displays definite caustic boundaries (Rines et al. 2003) up to a clustercentric radius of at least up to about $14 \mathrm{Mpc}$. If the Coma cluster is surrounded by an Einstein-Straus vacuole $r_{\mathrm{ta}}(\mathrm{Coma}) \approx 20 \mathrm{Mpc}$ is predicted $^{2}$, whereas the concordance model (Eq. (5)) predicts that $r_{\mathrm{ta}}(\mathrm{Coma}) \leq 13 \mathrm{Mpc}$.

Should further observations confirm the existence of Einstein-Straus vacuoles around a fraction of clusters of galaxies, this would indicate that at least one fundamental theoretical element is still missing in the present concordance model of cosmology. The most conservative (but not only) options would seem to be a vanishing $\Lambda$ or an appropriately modified form of dark energy.

\footnotetext{
${ }^{1}$ In principle e.g. modifications of GR could make hypothesis ES true, even with a concordance $\Lambda$.

${ }^{2}$ For a cluster mass of $2 \times 10^{15} M_{\odot}$.
} 
Acknowledgements. I thank Antonaldo Diaferio and Kenneth Rines for very helpful correspondence on the subject of this paper and reading a previous version of the manuscript. The constructive suggestions of an anonymous referee greatly improved the manuscript. Stacy McGaugh and Moti Milgrom patiently answered questions about the structure of spacetime in an expanding universe.

\section{References}

Bonnor, W. B. 1987, ApJ, 316, 49

Einstein, A., \& Straus, E. G. 1945, Rev. Mod. Phys., 17, 120

Einstein, A., \& Straus, E. G. 1946, Rev. Mod. Phys., 18, 148
Diaferio, A. 1999, MNRAS, 309, 610

Diaferio, A. 2005, private communication

Gilbert, C. 1956, MNRAS, 116, 678

Kaiser, N. 1987, MNRAS, 227, 1

Kolb, E. W., Matarrese, S., Notari, A., \& Riotto, A. 2005, Phys. Rev., D71, 023524

Rines, K., Geller, M. J., Kurtz, M. J., \& Diaferio, A. 2003, AJ, 126, 2152

Rines, K. 2005, private communication

Spergel, D. N., Verde, L., Peiris, H. V., et al. 2003, ApJS, 148, 175

Zwicky, F., \& Humason, M. L. 1964, ApJ, 139, 269 